Original paper

\title{
Comparative analysis of metabolic risk factors for progression of non-alcoholic fatty liver disease
}

\author{
Vincent J. H. Yao ${ }^{1}$, Michael Sun², Aivi A. Rahman', Zachariah Samuel', Joyce Chan ${ }^{3}$, Elizabeth Zheng ${ }^{4}$, Alan C. Yao ${ }^{5}$ \\ 'CUNY School of Medicine/Sophie Davis Biomedical Education Program, New York, USA \\ ${ }^{2}$ Cornell University, College of Agriculture and Life Sciences, Ithaca, USA \\ ${ }^{3} T$ he Chinese University of Hong Kong, Shatin, NT, Hong Kong SAR, The People's Republic of China \\ ${ }^{4}$ Columbia University Medical Center, New York, USA \\ ${ }^{5}$ Long Island Jewish Medical Center, Northwell Health, New York, USA
}

\begin{abstract}
Aim of the study: Non-alcoholic fatty liver disease (NAFLD), a globally prevailing chronic liver condition, refers to a spectrum of disease ranging from bland steatosis to steatohepatitis causing fibrosis without significant alcohol intake. Prominent risk factors (RFs) include obesity, type 2 diabetes mellitus, and dyslipidemia. Currently, no established hierarchy exists for the influence of metabolic RFs on NAFLD progression. This retrospective cohort study investigated and ranked the independent and combined effects of three major RFs on NAFLD progression.

Material and methods: 652 NAFLD patients with $\geq 1$ RF were categorized by RF combination to examine yearly changes in RF severity with liver stiffness measurement (LSM) over five years. Body mass index (BMI), hemoglobin $\mathrm{A}_{1 \mathrm{c}}\left(\mathrm{HbA}_{1 \mathrm{c}}\right)$, total cholesterol (TC), and LSM were reviewed.

Results: In patients with any single improving RF, decreases in BMI were associated with a yearly LSM change of $-1.26 \mathrm{kPa}$, while decreases in $\mathrm{HbA}_{1 \mathrm{c}}$ and $\mathrm{TC}$ were associated with a change of $-0.51 \mathrm{kPa}$ and $-0.56 \mathrm{kPa}$, respectively. In patients with any single worsening RF, increases in $\mathrm{BMI}$ were correlated with an $\mathrm{LSM}$ change of $+0.74 \mathrm{kPa}$ and increases in $\mathrm{HbA}_{1 \mathrm{c}}$ and $\mathrm{T}_{\mathrm{c}}$ were correlated with a change of $+0.43 \mathrm{kPa}$ and $+0.16 \mathrm{kPa}$, respectively. Patients with three RFs had the greatest LSM changes for both improving (-3.68 kPa) and worsening (+3.19 kPa) groups. The strongest predictors for $\mathrm{LSM}$ change were $\mathrm{BMI}$ and $\mathrm{HbA}_{1 \mathrm{c}^{\prime}}$ with standardized $\beta$ coefficients of 0.236 and $0.226(p<0.001)$, while TC had the least influence $[0.112(p<0.01), F(3,647)=11.458$, $\left.p<0.001, R^{2}=0.155\right]$.
\end{abstract}

Conclusions: Obesity was the most prominent RF. Treatment of all three RFs over a five-year period presented a high likelihood of fibrosis stage regression for NAFLD patients.

Key words: fibrosis, NAFLD, risk factor, transient elastography, liver stiffness measurement.

Address for correspondence:

Vincent Yao, CUNY School of Medicine/Sophie Davis Biomedical Education Program, 680 Bryant Ave, Roslyn, NY 11576, USA, e-mail: vya0328@gmail.com

\section{Introduction}

Non-alcoholic fatty liver disease (NAFLD) is currently the leading cause of chronic liver disease-related death. In 2019, NAFLD had an estimated global prevalence of 1.8 billion individuals [1]. This prevalence grows alongside the worldwide obesity epidemic, especially in the United States, with NAFLD affecting approximately
$25 \%$ of the U.S. population [2]. Non-alcoholic steatohepatitis (NASH), characterized by inflammation and hepatocellular injury, is the most severe form of NAFLD and affects nearly $20 \%$ of NAFLD patients [3]. Untreated, the progression of NAFLD can cause complications, including liver cancer and cirrhosis. It is expected that NAFLD will be the number one leading cause of liver transplants in the United States by 2025 [4]. Still, no approved pharmacologic therapy targeting the progres- 
sion of NAFLD is available, leaving lifestyle modifications and management of metabolic risk factors (RFs) the cornerstone of NAFLD treatment [5]. Substantial evidence suggests a bidirectional association between NAFLD and features of metabolic RFs, which are characterized by multiple medical conditions, the three most prevalent being obesity, type 2 diabetes mellitus (T2DM), and dyslipidemia $[1,6]$. An independent association was identified between the grade of liver steatosis as assessed by ultrasound and the risk of having these metabolic RFs [7].

Furthermore, these RFs may work synergistically to amplify NAFLD. Increases in both body mass index (BMI) and hemoglobin $\mathrm{A}_{1 c}\left(\mathrm{HbA}_{1 c}\right)$ have been found to correlate with greater insulin resistance (IR), which is a determining factor in the exacerbation of NAFLD $[8,9]$. Patients with T2DM experience a reduced ability to inhibit gluconeogenesis and exhibit increased rates of lipogenesis, which intensifies inflammation of the liver and increases the severity of IR [10]. Dyslipidemia may lead to progression of NAFLD through activation of inflammatory signaling pathways. Elevated cholesterol levels in the body activate hypoxia-inducible factor $1 a$ (HIF$1 \alpha$ ) and inducible nitric oxide synthase (iNOS), which then impair lipid metabolism [11]. Therefore, increases in IR caused by obesity and T2DM may work synergistically with decreases in lipid metabolism caused by dyslipidemia to worsen NAFLD.

While past studies explored management of metabolic RFs in patients, no prior research has compared the impacts of different combinations and severities of RFs on the progression of NAFLD. This study compared patients with one or more RFs to assess how the improvement or worsening of each RF or a combination of RFs can affect NAFLD progression. In doing so, we aimed to determine whether particular RFs should be prioritized in future treatment plans for NAFLD.

\section{Material and methods}

\section{Inclusion criteria}

Patients diagnosed with NAFLD on the basis of elevated alanine aminotransferase values $(\geq 40 \mathrm{IU} / \mathrm{ml}$ for men and $\geq 31 \mathrm{IU} / \mathrm{ml}$ for women) were included in this study. An ultrasound scan was also conducted on each patient to confirm the presence of fatty liver. Additionally, patients required the diagnosis of at least one of the following: obesity, T2DM, or dyslipidemia. Patients with a BMI of $\geq 30 \mathrm{~kg} / \mathrm{m}^{2}$ were classified as obese. Patients with a $\mathrm{HbA}_{1 \mathrm{c}}$ of $\geq 7.0 \mathrm{mmol} / \mathrm{l}$ were classified as type 2 diabetic. Patients with a total cholesterol (TC) level of $\geq 200 \mathrm{mg} / \mathrm{dl}$ were classified as dyslipidemic [12].

\section{Exclusion criteria}

We excluded patients under the age of 18 in this study. Those with a history of bariatric surgery or significant alcohol consumption (daily alcohol intake of $>20 \mathrm{~g}$ ), polycystic ovarian syndrome, and type 1 diabetes mellitus were also excluded. Other causes of underlying liver disease such as drug-induced liver disease, alcoholic liver disease, autoimmune hepatitis, primary biliary cirrhosis, a1-antitrypsin deficiency, hemochromatosis, Wilson's disease, biliary obstruction, and chronic hepatitis B and C were excluded as well.

\section{Study procedure}

Obese patients were instructed to adhere to a Mediterranean diet with a caloric deficit due to its efficacy in promoting weight loss and treatment of NAFLD [13]. Patients with T2DM were advised to decrease carbohydrate consumption. Patients with dyslipidemia were suggested to avoid high cholesterol foods. Both diabetic and dyslipidemic patients were advised to continue with pharmacological management. Lastly, all patients were advised to increase daily cardiovascular exercise and weight training as tolerated.

To analyze the potential compounding effects of metabolic RFs, patients were categorized into seven groups: obese, diabetic, dyslipidemic, obese and diabetic, obese and dyslipidemic, diabetic and dyslipidemic, and obese, diabetic, and dyslipidemic.

An Institutional Review Board was not available owing to the fact that this was a private practice facility. This review was of minimal risk to study participants given its retrospective nature. The electronic medical records of two gastrointestinal clinics were used for data collection. All data generated throughout this study were confidential. Patient names were converted into an identification code of numbers and letters. All data were password-protected and were only accessible in the clinics. $\mathrm{HbA}_{1 \mathrm{c}} \mathrm{TC}$, and liver stiffness measurement (LSM) values were collected from laboratory reports and transient elastography readings (with an M/XL probe). Transient elastography readings were obtained with FibroScan, manufactured by Echosens. According to the standard clinical protocol, each LSM measurement was performed in a fasting condition, confirmed after patients were told to fast 4 hours prior to examination. Patient age, gender, and BMI were collected during annual physical examinations. The stages of NAFLD patients were classified based on the F0, F1, F2, F3, and F4 parameters, which were $5.7 \pm 1.8 \mathrm{kPa}, 6.8 \pm 2.4 \mathrm{kPa}, 7.8 \pm 2.4 \mathrm{kPa}, 11.8 \pm 5.2 \mathrm{kPa}$, and $25.1 \pm 17.1 \mathrm{kPa}$, respectively [14]. Stages F0-F1 indicated minimal to mild fibrosis, stage F2 indicated moderate fibrosis, stage F3 indicated advanced fibrosis, and 
stage F4 indicated cirrhosis. A five-year range of BMI, $\mathrm{HbA}_{1 \mathrm{c}}$, and TC values was used to calculate the average and percent yearly change of RFs. These values were compared with the average yearly LSM change over five years. All patients had a minimum of one additional follow-up consultation in the five-year period, with follow-ups being at least one year apart. Despite efforts to keep patient followups exactly one year apart, patients sometimes had irregular followup intervals for certain years, resulting in varying times for their LSM measurements throughout each year.

\section{Statistical analyses}

A one-way ANOVA was used to assess the statistical significance of RFs in different RF combinations. Using SPSS v.25, both a Tukey post-hoc test and a multiple regression analysis were conducted. In the regression, the percent yearly changes in RFs were the predictor variables, while the average yearly changes in LSMs were the outcome variable. RFs with a $p<0.05$ were considered significant, and comparisons between the RFs were made using the standardized $\beta$ values. Multicollinearity of the predictor variables was ruled out, as assessed by the variance inflation factor.

\section{Results}

\section{Baseline characteristics}

Records for 4,609 patients were reviewed. Based on inclusion and exclusion criteria, 652 patients were in- cluded in the analysis. In the clinic population, 346 patients $(53.1 \%)$ had two follow-ups (mean time between visits: 1.54 years), 178 patients $(27.3 \%)$ had three follow-ups (mean time between visits: 1.28 years), 93 patients $(14.3 \%)$ had four follow-ups (mean time between visits: 1.11 years), and 35 patients (5.4\%) had five follow-ups over five years (mean time between visits: 1 year). Of the sample included, 345 were male (52.9\%) and 307 were female $(47.1 \%)$. Patients had a median of 56 years of age (range 18 to 89 years). The average BMI of obese patients was $35.28 \mathrm{~kg} / \mathrm{m}^{2}$. The average $\mathrm{HbA}_{1 \mathrm{c}}$ of patients with T2DM was $8.38 \mathrm{mmol} / \mathrm{l}$. The average TC of dyslipidemic patients was $230.77 \mathrm{mg} / \mathrm{dl}$ (Table 1).

\section{Multiple regression analysis}

Multiple linear regression was used to develop a model for analyzing average yearly changes in LSMs based on a patient's average yearly percent changes in BMI, TC, and $\mathrm{HbA}_{1 \mathrm{c}}$. As indicated by Table 2, the three-predictor model was able to account for $15.5 \%$ of the variance in the average change in LSMs, $\mathrm{F}(3,647)=11.458, p<0.001, R^{2}=0.155$. After standardizing the $\beta$ coefficients, variations in BMI predicted the greatest LSM changes, followed by $\mathrm{HbA}_{1 \mathrm{c}}$ and then TC. An increase of one standard deviation in BMI yielded an increase in 0.12 standard deviations in LSM $(p<0.001)$. An increase of one standard deviation in $\mathrm{HbA}_{1 \mathrm{c}}$ resulted in a similar increase of 0.09 standard deviations in LSM $(p<0.001)$. TC had the weakest effect on LSM, with an increase of one standard de-

Table 1. Average baseline characteristics among all patient groups

\begin{tabular}{lccccc}
\hline Patient group & $\boldsymbol{n}$ & Average LSM $(\mathbf{k P a})$ & Average BMI $\left(\mathbf{k g} / \mathbf{m}^{2}\right)$ & Average $\mathrm{HbA}_{1 \mathrm{c}}(\mathbf{m m o l} / \mathbf{l})$ & Average TC (mg/dl) \\
\hline Obesity only & 99 & 7.74 & 35.28 & $\mathrm{~N} / \mathrm{A}$ & $\mathrm{N} / \mathrm{A}$ \\
\hline T2DM only & 33 & 7.66 & $\mathrm{~N} / \mathrm{A}$ & 8.38 & $\mathrm{~N} / \mathrm{A}$ \\
\hline Dyslipidemia only & 193 & 5.74 & $\mathrm{~N} / \mathrm{A}$ & $\mathrm{N} / \mathrm{A}$ & 230.77 \\
\hline Obesity and T2DM only & 21 & 13.12 & 37.03 & 8.21 & $\mathrm{~N} / \mathrm{A}$ \\
\hline Obesity and dyslipidemia only & 130 & 6.39 & 34.57 & $\mathrm{~N} / \mathrm{A}$ & 225.53 \\
\hline T2DM and dyslipidemia only & 99 & 6.48 & $\mathrm{~N} / \mathrm{A}$ & 8.51 & 226.06 \\
\hline Obesity, T2DM, and dyslipidemia & 77 & 8.13 & 33.65 & 8.45 & 232.21 \\
\hline
\end{tabular}

LSM - liver stiffness measurement, BMI - body mass index, $H b A_{I C}$ - hemoglobin $A_{l l^{\prime}} T C$ - total cholesterol, T2DM - type 2 diabetes mellitus

Table 2. Effect of average yearly percent changes in NAFLD risk factors on average yearly changes in liver stiffness measurement (LSM)

\begin{tabular}{lcccc}
\hline Predictor & B & $\beta$ & $95 \%$ Cl (lower/upper) & $p$-value \\
\hline Average yearly percent change in body mass index & 0.12 & 0.24 & $(0.08,0.16)$ & $<0.001^{* * *}$ \\
\hline Average yearly percent change in total cholesterol & 0.03 & 0.11 & $(0.01,0.04)$ & $0.007^{* *}$ \\
\hline Average yearly percent change in hemoglobin $\mathrm{A}_{1 \mathrm{*}}$ & 0.09 & 0.23 & $(0.06,0.12)$ & $<0.001^{* * *}$ \\
\hline & & & & $R^{2}=0.155$ \\
\hline
\end{tabular}

${ }^{* *} p<0.01,{ }^{* * *} p<0.001$ 
Table 3. Average yearly changes in risk factor characteristic among all improving patient groups (2015-2019)

\begin{tabular}{|c|c|c|c|c|}
\hline Patient group & $n$ & $\begin{array}{l}\text { Average yearly BMI change } \\
\qquad\left(\mathrm{kg} / \mathrm{m}^{2}\right)\end{array}$ & $\begin{array}{l}\text { Average yearly } \mathrm{HbA}_{1 \mathrm{c}} \text { change } \\
(\mathrm{mmol} / \mathrm{l})\end{array}$ & $\begin{array}{l}\text { Average yearly TC change } \\
\text { (mg/dl) }\end{array}$ \\
\hline Obesity only & 47 & -3.04 & $\mathrm{~N} / \mathrm{A}$ & N/A \\
\hline T2DM only & 13 & $\mathrm{~N} / \mathrm{A}$ & -2.07 & $\mathrm{~N} / \mathrm{A}$ \\
\hline Dyslipidemia only & 96 & $\mathrm{~N} / \mathrm{A}$ & $\mathrm{N} / \mathrm{A}$ & -9.42 \\
\hline Obesity and T2DM only & 8 & -3.55 & -3.41 & $\mathrm{~N} / \mathrm{A}$ \\
\hline Obesity and dyslipidemia only & 68 & -2.54 & $\mathrm{~N} / \mathrm{A}$ & -7.33 \\
\hline T2DM and dyslipidemia only & 35 & $\mathrm{~N} / \mathrm{A}$ & -2.65 & -6.94 \\
\hline Obesity, T2DM, and dyslipidemia & 31 & -2.59 & -3.90 & -11.31 \\
\hline
\end{tabular}

$B M I$ - body mass index, $H b A_{I C}$ - hemoglobin $A_{I c^{\prime}} T C$ - total cholesterol, T2DM - type 2 diabetes mellitus

viation in TC producing an increase of 0.03 standard deviations in LSM $(p=0.007)$. Average yearly percent changes in $\mathrm{BMI}$ and $\mathrm{HbA}_{1 \mathrm{c}}$ had more than twice the impact on LSM changes compared to average yearly percent changes in TC.

\section{Patients with one risk factor}

When comparing patient groups with any single improving RF, reductions in BMI (Table 3) in obese patients had the greatest average yearly LSM change of $-1.26 \mathrm{kPa}$ (Fig. 1). A negative LSM change may suggest a regression in fibrosis. Reductions in TC (Table 3) in dyslipidemic patients had an average yearly LSM change of $-0.56 \mathrm{kPa}$ (Fig. 1). Lastly, reductions in $\mathrm{HbA}_{1 \mathrm{c}}$ (Table 3) in diabetic patients had the lowest average yearly LSM change of $-0.51 \mathrm{kPa}$ (Fig. 1). Obese patients yielded a $147 \%$ greater LSM change when compared to diabetic patients and a $125 \%$ greater LSM change when compared to dyslipidemic patients.

When comparing patient groups with any single worsening RF, increases in BMI (Table 4) in obese

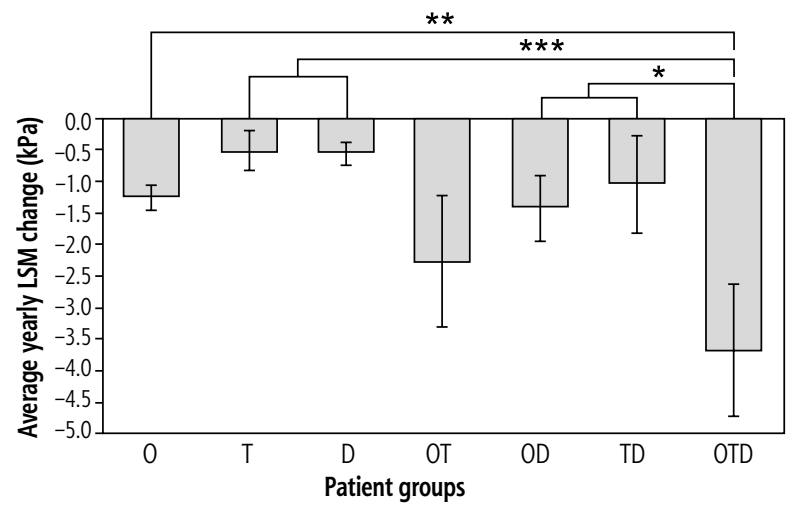

Fig. 1. Comparison of average yearly liver stiffness measurement (LSM) changes in improving patient groups (obese [0], type 2 diabetic [T], dyslipidemic [D], obese and type 2 diabetic [OT], obese and dyslipidemic [OD], type 2 diabetic and dyslipidemic [TD], and obese, type 2 diabetic, and dyslipidemic [OTD]), ${ }^{* *} p<0.01,{ }^{* *} p<0.001$ compared to OTD patients had the greatest average yearly LSM change of $+0.74 \mathrm{kPa}$ (Fig. 2). A positive LSM change suggests a progression of fibrosis. Increases in $\mathrm{HbA}_{1 \mathrm{c}}$ (Table 4) in diabetic patients had an average yearly LSM change of $+0.43 \mathrm{kPa}$ (Fig. 2). Finally, increases in TC (Table 4) in dyslipidemic patients had the smallest average yearly LSM change of $+0.16 \mathrm{kPa}$ (Fig. 2). Obese patients had a $72 \%$ greater LSM change when compared to diabetic patients and a 363\% greater LSM change when compared to dyslipidemic patients.

\section{Patients with two risk factors}

Upon comparing patient groups with any two improving RFs, reductions in both $\mathrm{BMI}$ and $\mathrm{HbA}_{1 \mathrm{c}}$ (Table 3 ) in obese and diabetic patients had the greatest average yearly LSM change of $-2.26 \mathrm{kPa}$ (Fig. 1). Reductions in both BMI and TC (Table 3) in obese and dyslipidemic patients had an average yearly LSM change of $-1.41 \mathrm{kPa}$ (Fig. 1). Lastly, reductions in both $\mathrm{HbA}_{1 \mathrm{c}}$ and TC (Table 3) in diabetic and dyslipidemic patients had the lowest average yearly LSM change of $-1.04 \mathrm{kPa}$ (Fig. 1). Obese

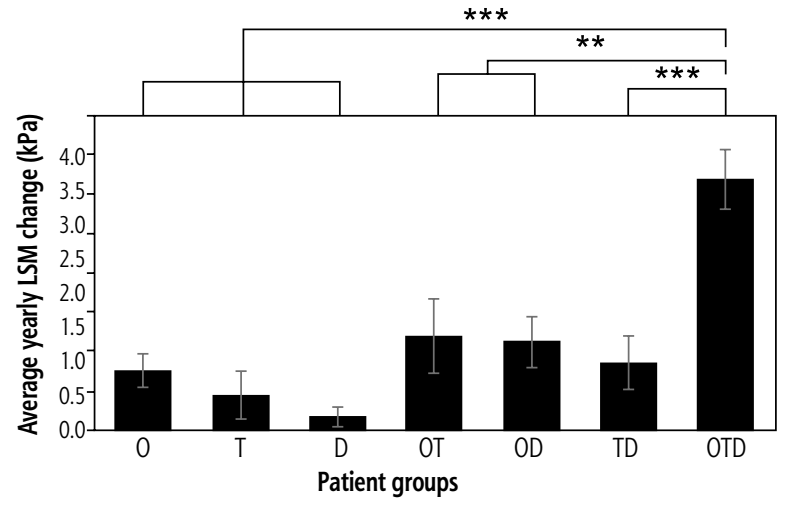

Fig. 2. Comparison of average yearly liver stiffness measurement (LSM) in changes worsening patient groups (obese [0], type 2 diabetic [T], dyslipidemic [D], obese and type 2 diabetic [OT], obese and dyslipidemic [OD], type 2 diabetic and dyslipidemic [TD], and obese, type 2 diabetic, and dyslipidemic [OTD]), ${ }^{* *} p<0.01,{ }^{* * *} p<0.001$ compared to OTD 
Table 4. Average yearly changes in RF characteristic among all worsening patient groups (2015-2019)

\begin{tabular}{|c|c|c|c|c|}
\hline Patient group & $n$ & $\begin{array}{l}\text { Average yearly BMI change } \\
\qquad\left(\mathrm{kg} / \mathrm{m}^{2}\right)\end{array}$ & $\begin{array}{l}\text { Average yearly } \mathrm{HbA}_{1 \mathrm{c}} \text { change } \\
(\mathrm{mmol} / \mathrm{l})\end{array}$ & $\begin{array}{l}\text { Average yearly TC change } \\
\text { (mg/dl) }\end{array}$ \\
\hline Obesity only & 52 & +3.64 & N/A & N/A \\
\hline T2DM only & 20 & N/A & +2.37 & N/A \\
\hline Dyslipidemia only & 97 & N/A & N/A & +9.87 \\
\hline Obesity and T2DM only & 13 & +2.61 & +2.30 & N/A \\
\hline Obesity and dyslipidemia only & 62 & +2.87 & N/A & +6.52 \\
\hline T2DM and dyslipidemia only & 64 & N/A & +3.92 & +6.10 \\
\hline Obesity, T2DM, and dyslipidemia & 46 & +3.51 & +3.25 & +10.97 \\
\hline
\end{tabular}

$B M I$ - body mass index, $H b A_{1 c}$ - hemoglobin $A_{l c} T C$ - total cholesterol, T2DM - type 2 diabetes mellitus

and diabetic patients had a $60 \%$ greater LSM change compared to obese and dyslipidemic patients and a $117 \%$ greater LSM change compared to diabetic and dyslipidemic patients. With the exception of improving diabetic and dyslipidemic patients, patient groups with any two improving RFs were observed to have greater average yearly LSM changes when compared to patient groups with any single improving RF (Fig. 1).

Upon comparing patient groups with any two worsening RFs, increases in both BMI and $\mathrm{HbA}_{1 c}$ (Table 4) in obese and diabetic patients had the greatest average yearly LSM change of $+1.19 \mathrm{kPa}$ (Fig. 2). Increases in both BMI and TC (Table 4) in obese and dyslipidemic patients had a similar average yearly LSM change of $+1.11 \mathrm{kPa}$ (Fig. 2). Finally, increases in both $\mathrm{HbA}_{1 \mathrm{c}}$ and TC (Table 4) in diabetic and dyslipidemic patients had the lowest average yearly LSM change of $+0.85 \mathrm{kPa}$ (Fig. 2). Obese and diabetic patients had a 7\% greater LSM change compared to obese and dyslipidemic patients and a $40 \%$ greater LSM change compared to diabetic and dyslipidemic patients. Patient groups with any two worsening RFs were found to have greater yearly LSM changes when compared to patient groups with any single worsening RF (Fig. 2).

\section{Patients with three risk factors}

Among all improving patient groups, reductions in BMI, $\mathrm{HbA}_{1 \mathrm{c}}$, and TC (Table 3) in obese, diabetic, and dyslipidemic patients yielded the greatest average yearly LSM change of $-3.68 \mathrm{kPa}$ (Fig. 1). Obese, diabetic, and dyslipidemic patients had a $192 \%$ greater LSM change compared to obese patients and a $63 \%$ larger LSM change compared to obese and diabetic patients. Obese, diabetic, and dyslipidemic patients had the most significant average yearly LSM change when compared to patient groups with any one $\mathrm{RF}(p<0.01)$ and patient groups with any two RFs $(p<0.05)$.
Among all worsening patient groups, increases in BMI, $\mathrm{HbA}_{1 \mathrm{c}}$, and TC (Table 4) in obese, diabetic, and dyslipidemic patients yielded the greatest average yearly LSM change of $+3.19 \mathrm{kPa}$ (Fig. 2). Obese, diabetic, and dyslipidemic patients had a $331 \%$ greater LSM change compared to obese patients and a $168 \%$ greater LSM change compared to obese and diabetic patients. Obese, diabetic, and dyslipidemic patients had the most significant average yearly LSM change when compared to patient groups with any one RF $(p<0.001)$ and patient groups with any two RFs $(p<0.01)$.

\section{Discussion}

\section{Summary of results}

In this retrospective cohort study, patients with different RF combinations were compared by assessing how changes in their RF severities affected NAFLD progression over a five-year period. When ranking the individual outcomes of RF changes, percent changes (positive or negative) in BMI were correlated with the highest magnitude of LSM change, followed by $\mathrm{HbA}_{1 c}$ and TC, respectively.

Reductions in both BMI and $\mathrm{HbA}_{1 c}$ in obese and diabetic patients were found to have the greatest effect on LSM change for improving patients with any two RFs. Patients with improved $\mathrm{HbA}_{1 \mathrm{c}}$ and $\mathrm{BMI}$ as well as patients with improved BMI and TC demonstrated a greater average yearly LSM change when compared to patients with any single improving $\mathrm{RF}$.

In contrast, increases in $\mathrm{BMI}$ and $\mathrm{HbA}_{\mathrm{lc}}$ in obese and diabetic patients were found to have the greatest effect on LSM change for worsening patients with any two RFs. Patients with any two worsening RFs were found to have a greater average yearly LSM change when compared to patients with any single worsening RF.

Among patients with all three RFs, reductions in BMI, $\mathrm{HbA}_{1 \mathrm{c}}$, and TC were found to have the greatest 
effect on LSM change when compared to all improving patient groups regardless of number of RFs. Similarly, increases in $\mathrm{BMI}, \mathrm{HbA}_{1 \mathrm{c}}$, and $\mathrm{TC}$ were also found to have the greatest effect on LSM change when compared to all worsening patient groups. BMI was present in the most influential patient groups for one, two, and three RFs, suggesting a strong relationship with NAFLD progression.

\section{Clinical implications}

Treatment of all three RFs provided a decrease of $3.68 \mathrm{kPa} /$ year in liver stiffness. This may suggest a high likelihood of fibrosis stage regression for NAFLD patients over a five-year period. Therefore, while treatment for obesity should be prioritized due to its strong influence regardless of other additional RFs, it is also important to simultaneously manage T2DM and dyslipidemia. Since the treatment modalities for T2DM and dyslipidemia act effectively on their respective conditions in isolation $[15,16]$ patients with all metabolic RFs may need more drastic measures to effectively treat these conditions, especially in the setting of NAFLD. Evidence for the management of dyslipidemia and T2DM suggests that supervised lifestyle modifications and pharmacologic therapy are more efficacious than pharmacologic therapy alone $[17,18]$. Thus, more education and awareness are needed for patients and physicians to create a more comprehensive management plan that addresses overall healthful living that can further enhance currently available treatments.

\section{Limitations}

While we covered prominent metabolic RFs such as obesity, T2DM, and dyslipidemia within our analysis, the lack of data regarding other metabolic RFs, such as arterial hypertension, is a major limitation. Future studies should incorporate these RFs into their analyses for a more comprehensive comparative analysis.

Although patients were instructed by their physician to follow up every year, not all patients did so. Therefore, respective RF data or LSMs for five consecutive years were not available in some patients within the sample population. The follow-up time varied between patients based on their number of follow-ups, and this was accounted for in the analysis. Secondly, two groups had limited sample sizes, namely the diabetic patients and the obese and diabetic patients. Furthermore, some patients received different medications for their respective conditions, which may have resulted in varying levels of NAFLD improvement.
Due to the restrictions of a clinical setting, adherence to lifestyle changes, such as the Mediterranean diet, could not be measured.

We acknowledge that lack of a liver biopsy is a major limitation. However, given that this is a retrospective review, this reflects current clinical data. Furthermore, liver biopsy is an invasive procedure and cannot be practically obtained for every patient [5]. Therefore, the use of FibroScan to monitor fibrosis stage reflects current clinical practice. FibroScan has also been shown to be a reliable marker for hepatic fibrosis, with approximately $5 \%$ of measures being classified as poorly reliable [14, 19]. A 2019 study assessing the association between LSM and various other factors found that the degree of histologically identified liver fibrosis predicted LSM [20]. Although the LSM change may be attributed to the regression of liver fibrosis, and thus support LSM's validity as a noninvasive alternative for liver biopsy, verification through liver biopsy was not obtained to support this hypothesis, and further research is required. Additionally, repeating LSM to monitor liver fibrosis severity can help to improve the overall accuracy of the FibroScan result [21].

\section{Conclusion and future study}

In summary, we found that BMI was the most important factor for patients with any single RF, and that patients with all three RFs experienced the most significant LSM change, regardless of improvement or worsening of all parameters. In the cohorts with the most significant changes in LSM, obesity was the only RF that was present across all groups, suggesting that BMI plays a significant role in NAFLD. Future research is needed to further elucidate the importance of obesity in NAFLD and the compounding effects of metabolic RFs on NAFLD progression. This knowledge can aid in the development of potential treatments that can effectively target all three RFs while emphasizing continued lifestyle interventions.

\section{Acknowledgements}

We would like to thank Dr. Allyson Weseley for her guidance on statistics within this paper. We would also like to show our gratitude to Kevin Liu for his assistance in coding for data analysis.

\section{Disclosure}

The authors declare no conflict of interest. 


\section{References}

1. Younossi Z, Anstee QM, Marietti M, et al. Global burden of NAFLD and NASH: trends, predictions, risk factors and prevention. Nat Rev Gastroenterol Hepatol 2018; 15: 11-20.

2. Bellentani S, Scaglioni F, Marino M, Bedogni G. Epidemiology of non-alcoholic fatty liver disease. Dig Dis 2010; 28: 155-161.

3. Estes C, Razavi H, Loomba R, et al. Modeling the epidemic of nonalcoholic fatty liver disease demonstrates an exponential increase in burden of disease. Hepatology 2018; 67: 123-133.

4. Wong RJ, Aguilar M, Cheung R, et al. Nonalcoholic steatohepatitis is the second leading etiology of liver disease among adults awaiting liver transplantation in the United States. Gastroenterology 2015; 148: 547-555.

5. Chalasani N, Younossi Z, Lavine JE, et al. The diagnosis and management of nonalcoholic fatty liver disease: practice guidance from the American Association for the Study of Liver Diseases. Hepatology 2018; 67: 328-357.

6. Younossi ZM, Koenig AB, Abdelatif D, et al. Global epidemiology of nonalcoholic fatty liver disease - meta-analytic assessment of prevalence, incidence, and outcomes. Hepatology 2016; 64: 73-84.

7. Mustapic S, Ziga S, Matic V, et al. Ultrasound grade of liver steatosis is independently associated with the risk of metabolic syndrome. Can J Gastroenterol Hepatol 2018; 2018: 8490242.

8. Bae JC, Suh S, Park SE, et al. Regular exercise is associated with a reduction in the risk of NAFLD and decreased liver enzymes in individuals with NAFLD independent of obesity in Korean adults. PLoS One 2012; 7: e46819.

9. Ota T, Takamura $T$, Kurita $S$, et al. Insulin resistance accelerates a dietary rat model of nonalcoholic steatohepatitis. Gastroenterology 2007; 132: 282-293.

10. Mikolasevic I, Milic S, Wensveen TT, et al. Nonalcoholic fatty liver disease - a multisystem disease? World J Gastroenterol 2016; 22: 9488.

11. Tirosh O. Hypoxic signaling and cholesterol lipotoxicity in fatty liver disease progression. Oxid Med Cell Longev 2018; 7: 1-15.

12. Kopin L, Lowenstein CJ. Dyslipidemia. Ann Intern Med 2017; 167: ITC81-96.

13. Kontogianni MD, Tileli N, Margariti A, et al. Adherence to the Mediterranean diet is associated with the severity of non-alcoholic fatty liver disease. Clin Nutr 2014; 33: 678-683.

14. Wong VW, Vergniol J, Wong GL, et al. Diagnosis of fibrosis and cirrhosis using liver stiffness measurement in nonalcoholic fatty liver disease. Hepatology 2009; 51: 454-462.

15. Wong VW. Current prevention and treatment options for NAFLD. Adv Exp Med Biol 2018; 1061: 149-157.

16. Gu Q. Prescription cholesterol-lowering medication use in adults aged 40 and over: United States, 2003-2012. US Department of Health and Human Services, Centers for Disease Control and Prevention, National Center for Health Statistics; 2014.

17. Párraga-Martínez I, Escobar-Rabadán F, Rabanales-Sotos J, et al. Efficacy of a combined strategy to improve low-density lipoprotein cholesterol control among patients with hypercholesterolemia: a randomized clinical trial. Rev Esp Cardiol (Engl Ed) 2018; 71: 33-41.

18. Johansen MY, MacDonald CS, Hansen KB, et al. Effect of an intensive lifestyle intervention on glycemic control in patients with type 2 diabetes: a randomized clinical trial. JAMA 2017; 318: 637-646.

19. Pang JX, Pradhan F, Zimmer S, et al. The feasibility and reliability of transient elastography using Fibroscan ${ }^{\circ}$ : a practice audit of 2335 examinations. Can J Gastroenterol Hepatol 2014; 28: 143-149.

20. Eddowes PJ, Sasso M, Allison M, et al. Accuracy of FibroScan controlled attenuation parameter and liver stiffness measurement in assessing steatosis and fibrosis in patients with nonalcoholic fatty liver disease. Gastroenterology 2019; 156: 1717-1730.

21. Chuah K, Lai L, Vethakkan SR, et al. Liver stiffness measurement in non-alcoholic fatty liver disease: Two is better than one. J Gastroenterol Hepatol 2020; 35: 1404-1411. 